\title{
Distinct Functional Modules for Discrete and Rhythmic Forelimb Movements in the Mouse Motor Cortex
}

\author{
(DRiichiro Hira, ${ }^{1,2}$ Shin-Ichiro Terada, ${ }^{1,3}$ Masashi Kondo, ${ }^{1,4}$ and Masanori Matsuzaki ${ }^{1,2}$ \\ ${ }^{1}$ Division of Brain Circuits, National Institute for Basic Biology, Myodaiji, Okazaki 444-8585, Japan, ${ }^{2}$ Department of Basic Biology, SOKENDAI, The \\ Graduate University for Advanced Studies, Okazaki 444-8585, Japan, ${ }^{3}$ Laboratory of Cell Recognition and Pattern Formation, Graduate School of \\ Biostudies, Kyoto University, Kyoto 606-8501, Japan, and ${ }^{4}$ Physical and Health Education, Graduate School of Education, the University of Tokyo, Tokyo \\ 113-0033, Japan
}

Movements of animals are composed of two fundamental dynamics: discrete and rhythmic movements. Although the movements with distinct dynamics are thought to be differently processed in the CNS, it is unclear how they are represented in the cerebral cortex. Here, we investigated the cortical representation of movement dynamics by developing prolonged transcranial optogenetic stimulation (pTOS) using awake, channelrhodopsin-2 transgenic mice. We found two domains that induced discrete forelimb movements in the forward and backward directions, and these sandwiched a domain that generated rhythmic forelimb movements. The forward discrete movement had an intrinsic velocity profile and the rhythmic movement had an intrinsic oscillation frequency. Each of the forward discrete and rhythmic domains possessed intracortical synaptic connections within its own domain, independently projected to the spinal cord, and weakened the neuronal activity and movement induction of the other domain. pTOS-induced movements were also classified as ethologically relevant movements. Forepaw-to-mouth movement was mapped in a part of the forward discrete domain, while locomotion-like movement was in a part of the rhythmic domain. Interestingly, photostimulation of the rhythmic domain resulted in a nonrhythmic, continuous lever-pull movement when a lever was present. The motor cortex possesses functional modules for distinct movement dynamics, and these can adapt to environmental constraints for purposeful movements.

Key words: channelrhodopsin; complex movement; motor cortex; mouse; photostimulation

\section{Significance Statement}

Animal behavior has discrete and rhythmic components, such as reaching and locomotion. It is unclear how these movements with distinct dynamics are represented in the cerebral cortex. We investigated the dynamics of movements induced by longduration transcranial photostimulation on the dorsal cortex of awake channelrhodopsin-2 transgenic mice. We found two domains causing forward and backward discrete forelimb movements and a domain for rhythmic forelimb movements. A domain for forward discrete movement and a domain for rhythmic movement mutually weakened neuronal activity and movement size. The photostimulation of the rhythmic domain also induced nonrhythmic, lever-pull movement, when the lever was present. Thus, the motor cortex has functional modules with distinct dynamics, and each module retains flexibility for adaptation to different environments.

\section{Introduction}

The emergence of a variety of complex movements, such as skilled forelimb movements and instrument manipulation, is one

Received July 18, 2015; revised Aug. 16, 2015; accepted Aug. 20, 2015.

Author contributions: R.H. and M.M. designed research; R.H., S.-I.T., and M.K. performed research; R.H., S.-I.T., and M.M. contributed unpublished reagents/analytic tools; R.H., S.-I.T., and M.K. analyzed data; R.H. and M.M. wrote the paper.

This work was supported by Japan Science and Technology Agency, CREST (to M.M.), Scientific Research (Grants No. 23300148 and No. 15 H02350 to M.M.), by Scientific Research on Innovative Areas "Mesoscopic Neurocircuitry" (Grant No. 22115005 to M.M.), by Brain Mapping by Integrated Neurotechnologies for Disease Studies (to M.M.), by a Japan Society for the Promotion of Science Research Fellowship for Young Scientists (to S.-I.T.), by Grants-in-Aid for Young Scientists (№. 26830021 to R.H.), and a Takeda Foundation grant to M.M. We thank Ms. J. Saito, M. Himeno, of the amazing accomplishments of biological systems (Georgopoulos and Grillner, 1989; Iwaniuk and Whishaw, 2000; Hogan and Sternad, 2007; Shenoy et al., 2013). Complex movements are composed of two fundamental dynamics: discrete movements and rhythmic movements (Schaal et al., 2004; Hogan and Ster-

and S. Ohsawa for technical assistance; Drs. M. J. Choo, M. Morishima, Y. Ueta, Y.R. Tanaka, and A. Saiki for discussion; and Drs. Y. Isomura and H. Nishimaru for helpful comments on the manuscript.

The authors declare no competing financial interests.

Correspondence should be addressed to Prof. Masanori Matsuzaki, Division of Brain Circuits, National Institute for Basic Biology, Myodaiji, 0kazaki 444-8585, Japan. E-mail: mzakim@nibb.ac.jp.

DOI:10.1523/JNEUROSCI.2731-15.2015

Copyright $\odot 2015$ the authors $\quad 0270-6474 / 15 / 3513311-12 \$ 15.00 / 0$ 
nad, 2007; Degallier and Ijspeert, 2010; Howard et al., 2011). Discrete movements, such as reaching, are defined as continuous movements from a start point to an end point, whereas rhythmic movements, including locomotion, are defined as repetitive, oscillatory movements. In mammals, reaching and locomotion are driven by spinal subcircuits, which are thought to be modulated by the motor cortex (Beloozerova and Sirota, 1993; Alstermark and Isa, 2012). In fact, the cortical representation of reaching has been clarified in rodents and primates by studies using longduration intracortical microstimulation or optogenetic stimulation (Stepniewska et al., 2005; Graziano, 2006; Ramanathan et al., 2006; Harrison et al., 2012; Bonazzi et al., 2013; Brown and Teskey, 2014). However, rhythmic limb movements have not been mapped by the stimulation of the rodent or primate cortex. In humans, when discrete and rhythmic hand movements are volitionally executed, different motor cortical areas are activated (Schaal et al., 2004). However, it is unknown how these different cortical areas interact with each other.

Here, we use long-duration photostimulation in awake, headrestrained channelrhodopsin-2 (ChR2) transgenic mice (Ayling et al., 2009; Hira et al., 2009, 2013a,b) to demonstrate that discrete and rhythmic forelimb movements are generated in distinct cortical regions (domains). We also show that three types of ethologically relevant movements (Graziano, 2006) and lever-push/ pull movements (Hira et al., 2013a; Masamizu et al., 2014) are mapped in these distinct domains.

\section{Materials and Methods}

Animals. All experiments were approved by the Institutional Animal Care and Use Committee of National Institutes of Natural Sciences, Japan. Thy1-ChR2 transgenic mice (line 9, male and female, age 5-13 months) and wild-type mice (male, age 2-4 months) were used. Mice were anesthetized by intraperitoneal injection of ketamine $(74 \mathrm{mg} / \mathrm{kg})$ and xylazine $(10 \mathrm{mg} / \mathrm{kg})$, and an incision was made to the skin covering the neocortex. After the exposed skull was cleaned, a head plate was attached to the skull using dental cement (Fujiryu-to BC, GC; Bistite II, Tokuyama Dental). The surface of the intact skull was subsequently coated with dental adhesive resin cement based on acrylic resin (Super Bond, Sun Medical) to prevent drying.

Optogenetic stimulation. Mice were accustomed to a head-fixed condition with the body in the body chamber for $\geq 1 \mathrm{~d}$ before the photostimulation experiments. The mice were relaxed in the body chamber and their forelimbs were hanging free. Photostimulation experiments were performed using an upright microscope (BX61WI, Olympus) and an FV1000-MPE laser-scanning microscope system (Olympus). The laser beam was focused on the surface of the targeted cortical site via the microscope objective lens (MPlan $5 \times$, numerical aperture of 0.10 ; or PlanApo $2 \times$, numerical aperture of 0.08 ; Olympus). The surface of the resin coat was covered by a mixture of mineral oil $(50 \%)$ and petrolatum $(50 \%)$ to maximize its transparency. Laser power $(1.2-7.2 \mathrm{~mW}$ at the specimen plane) and single-pulse duration ( 2 or $4 \mathrm{~ms}$ ) were adjusted to a level that induced stable and visible responses in every experiment. For each site, 25 pulses were illuminated at $50 \mathrm{~Hz}$ for $500 \mathrm{~ms}$. To obtain trajectories of the right forepaw in response to stimulation of broad cortical areas, a $6 \times 3 \mathrm{~mm}$ left dorsal cortical area was divided into $16 \times$ 8 points and these points were pseudorandomly stimulated (Fig. $1 \mathrm{~A}$ ). The interstimulation interval was 1 or $1.5 \mathrm{~s}$. Data for maps were averaged over 20 cycles to cancel the effects of spontaneous movements.

For simultaneous photostimulation of two sites, the laser beam was equally split by a half mirror under the objective lens and the reflected laser beam was reflected by another mirror so that two beams with the same power $(1.7 \mathrm{~mW})$ were focused on the two target sites. The movements evoked by the split laser were indistinguishable from those evoked by the nonsplit laser focused on the same sites.

Three-dimensional tracking of the right forepaw. The right forepaw was painted white and the other body parts that have high reflection were painted black with enamel coating (white, X-2; black; XF-1; Tamiya). Two machine vision cameras (Scout scA640-70gm, Basler) equipped with fixed-focus lenses (focal length, $8 \mathrm{~mm}$; M0814-MP, Computer) were orthogonally set $100 \mathrm{~mm}$ from the right side of the mouse and 100 $\mathrm{mm}$ in front of the mouse (Fig. $1 B$ ) to track the position of the right forepaw during photostimulation. The two cameras were controlled by the LabView Vision module (National Instruments). Two images were simultaneously obtained at 200 frames per second using $4 \times 4$ binning $(164 \times 122$ binned pixels, $0.37 \mathrm{~mm}$ per pixel) by the two cameras. Exposure time was set at $0.5 \mathrm{~ms}$. Visible light that bifurcated into four was used as the light source. Each image was converted into a binary image in which the pixels that included a whitened forepaw were 1 and the other pixels were 0 . Then the center of mass of the forepaw was calculated from the binary image in real time. The vertical $(z)$ position of the right forepaw was obtained by both cameras, and the value used in analysis was calculated by averaging the values from the two cameras. The forwardbackward $(y)$ and medial-lateral $(x)$ positions were obtained by the sideview camera and the front-view camera, respectively. The position of the right forepaw at the prolonged transcranial optogenetic stimulation (pTOS) initiation is referred to as the initial point of the forepaw and the average position of the right forepaw $450-500 \mathrm{~ms}$ after the pTOS initiation is referred to the end point of the forepaw.

Estimation of trial-to-trial variability of the direction of the forelimb movement induced by pTOS. When any one of the $x, y$, or $z$ lengths of the vectors from the initial point of the forepaw trajectory to the end point of the forepaw trajectory over the 20 trials (20 movement vectors) for a given pTOS site was significantly larger than the corresponding lengths when the bone outside the cortex was photostimulated 20 times $(p<$ $0.05 / 3$, Wilcoxon's rank sum test), the pTOS site was defined as a "movement-related site." Eighty-one percent of all pTOS sites were movement related. To estimate the trial-to-trial variability of movement directions, the angle between each movement vector and the mean movement vector was calculated for each movement-related site. The mode of the angles for all movement-related sites was $21^{\circ}$.

Definitions of discreteness, straightness, rhythmicity, and rotation indices. Mathematically, discrete and rhythmic movements are referred to as aperiodic and periodic movements, respectively (Degallier and Ijspeert, 2010). However, when we consider natural forelimb movements, discrete movements often have straight trajectories with velocity curves with a single peak, whereas rhythmic movements have circular or back-and-forth trajectories. To quantify discrete movements, the average tangential speed at each time point was calculated using values from two adjacent time points, which had an interval of $5 \mathrm{~ms}$. Then, a Gaussian function with peak amplitude and peak center position that corresponded to the peak speed and peak center position of the original data was fitted to the data. Then, the coefficient of determination $R^{2}$ was obtained, defined as the discreteness index. To characterize the orbit, the distance between the initial and end points of the trajectory $\left(R_{0}\right)$ was divided by the total path length of trajectory during pTOS $\left(R_{\text {path }}\right)$. This was defined as the straightness index.

To quantify rhythmic movements, the frequency with the maximum power of the movements was calculated for the three axes and averaged. The rhythmicity index was defined as the average of the power from 5 to $15 \mathrm{~Hz}$ for each of the three axes during the $500 \mathrm{~ms}$ photostimulation. To estimate the rotation direction of rhythmic movements, the angle between the direction of movement at one time point and that at the next time point in the plane recorded by the right-side-view camera was summed across a trial. The minimum and maximum sum for the 20 trials performed in each mouse was averaged for forward and backward rotations, respectively. Thus, negative and positive values indicated forward (clockwise) and backward (anticlockwise) rotations of circular movements, respectively. This value was defined as the rotation index.

Analysis of ethologically relevant movements. Complex behaviors consistently evoked for $500 \mathrm{~ms}$ after the onset of the photostimulation were visually categorized as forepaw-to-mouth movements, locomotion-like movements, defensive-like movements, or other movements. When the contralateral forepaw moved toward the mouth and the mouth simultaneously opened, it was defined as a forepaw-to-mouth movement, which might correspond to hand-to-mouth movements in primates and bring- 
A
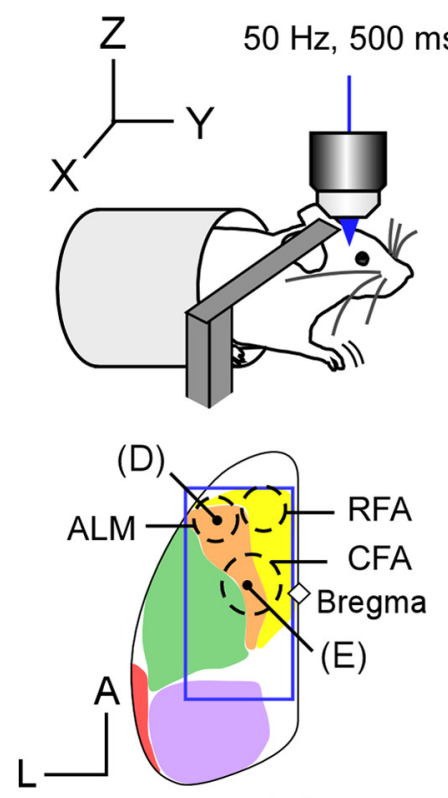

B
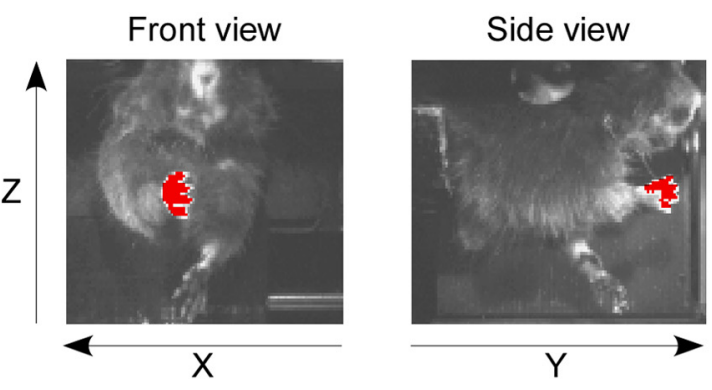

C

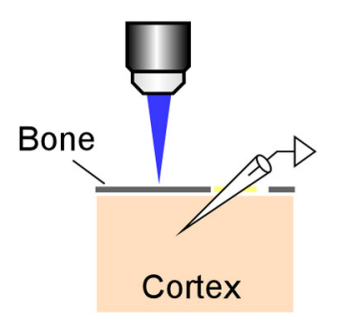

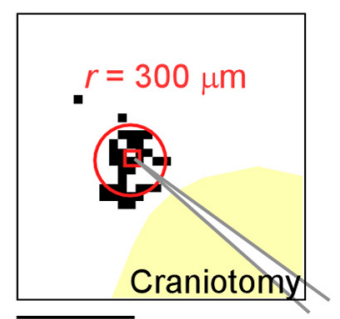

D
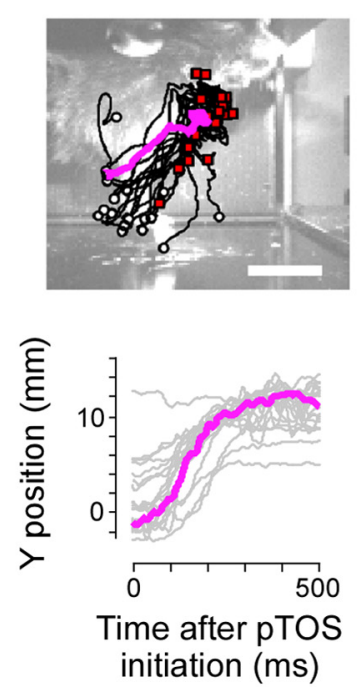

E
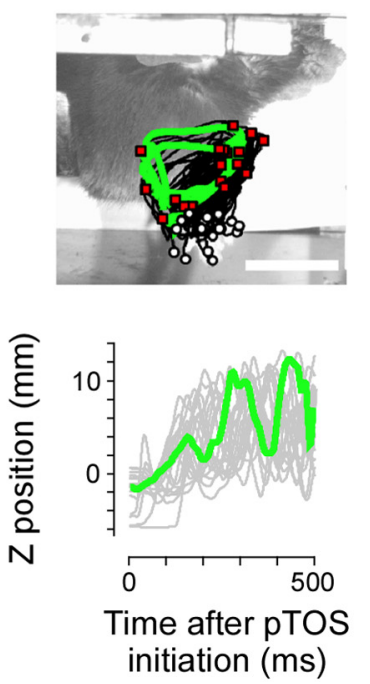

$\mathbf{F}$
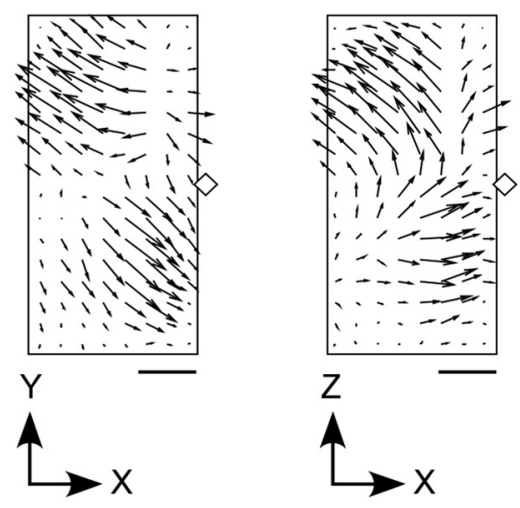

Z

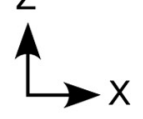

Figure 1. Discrete and rhythmic movements induced by pTOS. A, Top, Experimental setup for pTOS of an awake, head-restrained, ChR2 transgenic mouse. Bottom, Dorsal view of the left cerebral cortex. The blue square indicates the area over which maps were created. Colors indicate conventional functional regions: orange, lateral agranular cortex; yellow, medial agranular cortex; green, sensory cortex; purple, visual cortex; red, auditory cortex (modified from Kirkcaldie, 2012). Forelimb-related motor areas (ALM, RFA, and CFA) are schematically shown as dotted circles, based on Komiyama et al. (2010), Tennant et al. (2011), and Hira et al. (2013a,b). Black points indicate the pTOS sites shown in $\boldsymbol{D}$ and $\boldsymbol{E}$. The diamond indicates the bregma. Scale bar, $1 \mathrm{~mm}$. $\boldsymbol{B}$, Examples of simultaneous snapshots from two machine vision cameras. A front-view camera (left) and a side-view camera (right) were used to determine the right-forepaw position (red) along the $x$-axis and $z$-axis and along the $y$-axis and $z$-axis, respectively. C, Left, Schematic of the photostimulation experiment performed to estimate the resolution of transcranial photostimulation (horizontal view). The electrode was inserted into the cortex at a depth of $700 \mu \mathrm{m}$ from the surface of the brain and the evoked spiking activities were detected. Right, Example of the map obtained by the experiment illustrated in the left panel. Black dots indicate the locations of photostimuli that induced action potentials with a latency $\leq 1 \mathrm{~ms}$. The radius ( $r$ ) of the map is $\sim 300 \mu \mathrm{m}$ (Hira et al., 2009 , $2013 \mathrm{~b}$; Asrican et al., 2013). The yellow area denotes the area where the skull was removed. Scale bar, $1 \mathrm{~mm}$. D, E, Top, Images of a mouse obtained from the right side, overlaid on which are 20 right-forepaw trajectories induced by pTOS of the labeled site in $A$. White circles indicate the initial point of the forepaw. Red squares indicate the endpoint of the forepaw. Magenta and green indicate representative trajectories. Other trajectories are shown in black. Scale bar, $10 \mathrm{~mm}$. Bottom, Trajectories of the right forepaw in the top panels. $F$, Maps showing the direction and distance of the movement elicited by each pTOS site within the square shown in $A$. For each pTOS site, the direction of the movement and the distance of the movement along each axis are represented by the direction and length of the arrow, respectively. The distance of the movement was normalized in each mouse and averaged across three mice. The diamond indicates the bregma. Scale bar, $1 \mathrm{~mm}$.

to-body movements in rat (Bonazzi et al., 2013). When the contralateral forepaw was elevated with pronation and then moved outward and rhythmically shaken as if the mouse rejected something approaching the face, it was defined as a defensive-like movement. A human-audible squeak, which is emitted when the animal undergoes a stressful experience, and cheek movements often accompanied a defensive-like movement (Graziano et al., 2002; Bartolomucci et al., 2004). This might be similar to the "defensive" movement in primates (Cooke and Graziano, 2004). Bilateral, alternate forelimb movements were defined as a locomotion-like movement. This might be similar to the climbing movement in primates (Graziano et al., 2002). The hindlimbs also moved alternately in some trials, but movement of the hind limbs was not included in the criteria for locomotion-like movement. These movements were captured by the video camera (Everio, JVC; 30 frames/s) or machine 
vision camera described above and analyzed after the experiments. Movements of the contralateral digits (grasping), uncategorized contralateral forelimb movements, tongue movements (licking), and jaw movements were also identified by off-line eye inspection. A squeak was identified from the audio recording. Ultrasonic vocalizations and movements of the eyes, whiskers, hindlimbs, or tail were not quantified.

These experiments and the categorization of complex movements were performed by experimenters who did not have any knowledge of the maps of the discrete and rhythmic movements or the lever-push/pull movements.

Lever-push and lever-pull movements. Mice that were accustomed to the head-fixed condition and had not had any lever-movement training were used for this experiment (Hira et al., 2013a). The lever was located at the position where the head-restrained mouse naturally put the right forepaw. An immovable small grid was located at a position that allowed the left forelimb to rest on the grid. It was visually confirmed that the left forelimb or other body parts did not push or pull the lever. The maps for lever-push or lever-pull movements were obtained in separate experiments. When the lever-push movement was investigated by pTOS, the lever could not be pulled. When the lever-pull movement was investigated, the lever could not be pushed. In both experiments, the lever could be only horizontally moved by $5 \mathrm{~mm}$. For mapping, a $7.5 \times 2.5 \mathrm{~mm}$ area was divided into three $2.5 \times 2.5 \mathrm{~mm}$ areas and each area was divided into $8 \times 8$ sites. These sites were pseudorandomly stimulated with an interstimulus interval of $1.5 \mathrm{~s}$. Maps were created from an average of 20 stimuli at each site. The lever position was detected using a magnetic bead attached to the lever and a magnetic sensor located close by, as described in our previous studies (Hira et al., 2013a; Masamizu et al., 2014).

The lever movement was quantified as follows: first, the lever position at each time point was normalized between 0 (resting position) and 1 (fully pushed or pulled) as $L$. For each pTOS trial, the normalized lever position at the initiation of pTOS was set as $L_{\text {initial }}$. Non-negative values of $\left(L-L_{\text {initial }}\right)$ during $500 \mathrm{~ms}$ pTOS were averaged and the averaged value was divided by $1-L_{\text {initial }}$. Then, the value averaged across trials was defined as the lever-pushing or lever-pulling rate for each pTOS site. This rate ranged between 0 and 1 . To calculate the duration of the lever push or pull, pTOS sites with the top five lever-push or lever-pull rates in each mapping trial were selected in each mouse $(n=5$ sites $\times 20$ trials $\times 4$ mice $=400$ pTOS sites). Then, trials in which the maximum of $L-L_{\text {initial }}$ during pTOS exceeded a threshold, $0.2 \times\left(1-L_{\text {initial }}\right)$, were selected $(216$ and 346 trials for lever pushing and pulling, respectively). For each of the selected trials, the time to exceed the threshold during pTOS was summed.

Electrophysiological recordings and stimuli. Electrical recordings were conducted as previously described (Hira et al., 2013b). Briefly, tungsten microelectrodes with an impedance of 0.5-2 M $\Omega$ (TM33B20KT, WPI) were inserted into the anterior lateral part of the motor cortex (ALM), the caudal forelimb area (CFA), or the rostral forelimb area (RFA) in isoflurane-anesthetized $(0.7-1.1 \%)$ mice. The signals were amplified (DAM-80 amplifier, WPI), filtered at $300-1000 \mathrm{~Hz}$, and sampled at 5 $\mathrm{kHz}$ (FV1000 system, Olympus). A spike was detected when the current was lower than the threshold (2.5 SD below baseline mean).

Electrical stimuli were delivered by tungsten microelectrodes with an impedance of $2 \mathrm{M} \Omega$ (TM33B20KT, WPI), which were placed in the ALM, the CFA, or the mesencephalic locomotor region (MLR). The duration, frequency, and number of pulses were $200 \mu \mathrm{s}, 200 \mathrm{~Hz}$, and 100, respectively. The current intensity was slightly larger than the threshold to induce forelimb movements $(8-96 \mu \mathrm{A})$ at each stimulation site. The depth of the cortical stimulation was $600-800 \mu \mathrm{m}$ from the surface of the brain. MLR was stimulated by an electrode inserted at a site $4.5 \mathrm{~mm}$ posterior and $1.2 \mathrm{~mm}$ lateral to bregma and $3.5 \mathrm{~mm}$ ventral from the cortical surface (Lee et al., 2014). To determine the number of steps, a movement of the right forepaw from the highest to the lowest position was counted as a one step.

Anatomical tracing. EGFP and tdTomato were introduced into cortical neurons in wild-type mice by intracortical injection of AAV1-CaMKII$\operatorname{EGFP}\left(2.06 \times 10^{13}\right.$ vector genomes $\left./ \mathrm{ml}\right)$ and AAV1-CAG-tdTomato $\left(1.19 \times 10^{13}\right.$ vector genomes $\left./ \mathrm{ml}\right)$, respectively, which were provided by the University of Pennsylvania Gene Therapy Program Vector Core.
Mice were anesthetized with isoflurane $(0.8-1.1 \%$ for induction and maintenance) and then $0.25 \mu \mathrm{l}$ of each virus solution was injected at depths of 900 and $400 \mu \mathrm{m}$ below the pia in the ALM (2.4 mm anterior and $1.8 \mathrm{~mm}$ lateral to bregma) or the CFA $(0.2 \mathrm{~mm}$ anterior and $1.3 \mathrm{~mm}$ lateral to bregma) through a glass pipette (20-30 $\mu \mathrm{m}$ open tip diameter). The mice were then returned to their home cage. Three weeks after the injection, the mice were deeply anesthetized with ketamine $(74 \mathrm{mg} / \mathrm{kg})$ and xylazine $(10 \mathrm{mg} / \mathrm{kg})$ and transcardially perfused with $40 \mathrm{ml}$ of PBS and $40 \mathrm{ml}$ of $4 \%$ paraformaldehyde in PBS (Wako). The brains were removed and postfixed with the same fixative for $\geq 12 \mathrm{~h}$ at $4^{\circ} \mathrm{C}$. The brains were cut into coronal sections with a thickness of $50 \mu \mathrm{m}$. After staining the cell nuclei with DAPI, the slices were mounted on glass slides and overlaid with coverslips. Fluorescence was visualized through a fluorescence microscope with optical sectioning (BZ-X700, Keyence).

Cluster analysis using an anatomical database. The Allen Mouse Brain Connectivity Atlas (http://connectivity.brain-map.org; Oh et al., 2014) was used for clustering corticocortical synaptic interactions. In this database, connectivity data are available for mice from injection areas (AAV1-pSynI-EGFP as an anterograde tracer) to any brain region. We used Spatial Search mode and limited the injection sites to the isocortex of wild-type C57BL6J mice. We set a grid that covered wide dorsal cortical areas, including the frontal and parietal cortex $(5.8 \times 3.1 \mathrm{~mm}, 100$ $\mu \mathrm{m}$ apart, total 1821 sites). Then, the injection sites that project to each point in the grid were identified. Using these data, we obtained a binary connectivity matrix from 125 input sites to 1821 output sites. The IDs of the 125 injection sites are as follows: 100140756, 100140949, 100141219, $100141273,100141454,100141473,100141495,100141563,100141599$, 100141780, 100141796, 100142655, 100147853, 100148142, 100148503, 100149109, 100149969, 112162251, 112229103, 112229814, 112306316, 112373124, 112373830, 112423392, 112424813, 112458114, 112514202, 112514915, 112595376, 112596790, 112670853, 112791318, 112881858, 112882565, 112935169, 112936582, 112951804, 112952510, 113036264, 113887162, 113887868, 114250546, 114290938, 114292355, 115958825, 116903230, 116903968, 117298988, 120437703, 120491896, 126852363, 126860974, 126861679, 126862385, 126907302, 126908007, 126909424, 127084296, 127089669, 127138787, 127866392, 139426984, 139519496, 139520203, 141602484, 141603190, 142656218, 146077302, 146593590, 146858006, 146858755, 148964212, 157062358, 157556400, 157654817, 157710335, 157711748, 158314278, 158435116, 170721670, 174360333, 174361040, 174361746, 180073473, 180074890, 180296424, 180403712, 180435652, 180673746, 180709230, 180709942, 180717881, 180718587, 180719293, 180720175, 180916954, 180917660, 272414403, 272697944, 272737914, 272782668, 272916202, 272916915, 277616630, 277712166, 277713580, 277714322, 304564721, 304565427, 304585910, 304586645, 307137980, 307295727, 307296433, 307297141, 307320960, 307321674, 307557934, 307558646, 307593747, 307743253, 309003780, 309004492, 309113907 , and 309372716.

The data matrix was then used to conduct $K$-means clustering $(K=3)$ based on the correlation coefficient. For synaptic input clustering, the cortical sites were divided into three domains sharing similar synaptic input patterns. Likewise, for synaptic output clustering, the injection sites were divided into three groups sharing similar synaptic output patterns. Although the result of $K$-means clustering generally depends on initial random values, when the number of clusters $(K)$ was set at 3 , the clustering results were always the same for synaptic input clustering ( $100 \%$ sites were categorized to the same group in 100 iterations) and were highly robust for synaptic output clustering $(97.0 \%$ sites were categorized to the same group in 100 iterations).

Pharmacology. A solution containing $125 \mathrm{~mm} \mathrm{NaCl}, 4 \mathrm{~mm} \mathrm{KCl,} 5 \mathrm{~mm}$ HEPES, 2 mM CNQX (a competitive AMPA/kainite receptor antagonist; Tocris Bioscience), and $2 \mathrm{mM}$ D-AP5 (a competitive NMDA receptor antagonist; Sigma-Aldrich) was directly applied to the cortical surface after the skull and dura were removed. Photostimulation was performed immediately before and $30 \mathrm{~min}$ to $2 \mathrm{~h}$ after application of this solution and the induced movements were recorded. A similar procedure without D-AP5 completely blocked corticocortical synaptic inputs (Hira et al., 2013b) and these inhibitory effects were confirmed in additional electrophysiological experiments. 
Statistics. Data are expressed as mean \pm SD. The error bars in the graphs represent the SEM. The Wilcoxon signed-rank test was used for pairwise comparisons. ANOVA and post hoc Tukey's HSD test were used for multiple comparisons. No statistical tests were run to predetermine sample size. Blinding and randomization were not performed.

Confidence intervals of the optimal frequency for the induction of movements (see Fig. $3 B$ ) were estimated by bootstrapping as follows: a fourth-order polynomial regression model was used to fit the data and the estimated values and the residuals were calculated for every data point. Then, the data were resampled by randomly adding the residuals to the estimated variables. The fourth-order polynomial regression was conducted for the resampled dataset 10,000 times, and the optimal frequency was estimated in each dataset. Finally, the 250th largest and 250th smallest values from the 10,000 estimated optimal frequency values were determined as the upper and the lower limit of the $95 \%$ confidence intervals, respectively. Qualitatively similar results were obtained when the third-order and fifth-order polynomial regression models were used.

\section{Results}

\section{pTOS of the motor cortex in awake ChR2 mice induces}

\section{dynamic forelimb movements}

We examined the dynamics of complex movements of freely movable forelimbs in awake ChR2 transgenic mice with the skull coated by transparent resin (Fig. 1A; Hira et al., 2009). The left cortical surface was illuminated by a blue laser ( $500 \mathrm{~ms}$ train of 2 or $4 \mathrm{~ms}$ pulses delivered at $50 \mathrm{~Hz}$; Fig. 1A) focused through the objective lens. We call this method pTOS. The pTOS-induced trajectory of the right forepaw was tracked in three-dimensional space by two high-speed cameras at 100 or 200 frames/s (Fig. 1B). The lateral width of the cortical area exhibiting pTOS-induced neuronal firing was $\sim 600 \mu \mathrm{m}$ (Fig. $1 C$; Hira et al., 2009).

pTOS induced one of two major types of right-forelimb movement, depending on the stimulation site. During pTOS of the ALM, the right forepaw moved to a narrow space in every trial (Fig. $1 A, D$ ). By contrast, during pTOS of the CFA, the right forepaw moved circularly for $>1$ cycle and its end point varied (Fig. 1A,E). Either movement was not followed by apparent seizure-like movement after the end of pTOS. We categorize the former right-forelimb movement as a discrete movement, and the latter as a rhythmic movement.

To map the representation of the discrete and rhythmic forelimb movements, we divided a $6 \times 3 \mathrm{~mm}$ area of the left dorsal cerebral cortex into $16 \times 8$ sites (intersite distance of $400 \mu \mathrm{m}$ ) and performed pTOS at each site. The pTOS mapping was conducted 20 times in each of three animals. For the first analysis, the direction and distance from the mean initial point to the mean end point of the forepaw was calculated for each pTOS site. The direction and distance had clear topography in the cortex (Fig. $1 F)$. For each pTOS site that induced movements that were significantly larger than spontaneous movements, the angle between the movement direction in each trial and the mean movement direction over all 20 trials was calculated. The mode difference over all 20 trials from those pTOS sites (81\% of all pTOS sites from three mice) was $21^{\circ}$. Thus, long arrows on the map indicate the sites that induced forelimb movements with a limited target space and long distance. The ALM and the hindlimb motor area (HA), which is caudal to the CFA, had long arrows with almost opposite directions (Fig. 1F, left).

\section{pTOS resolves distinct domains for discrete and}

\section{rhythmic movements}

Next, we quantitatively estimated the discrete movement. The velocity profile of discrete movements had a bell-shaped curve (Fig. 2A), which is similar to the velocity profile of natural reach- ing movements (Morasso, 1981; Graziano et al., 2002). Therefore, for each pTOS site, the tangential velocity profile of the averaged movement was fitted with a Gaussian function and the coefficient of determination was defined as the "discreteness index." The domains with a large discreteness index (>0.7; Fig. $2 B$ ) corresponded to the ALM and the HA, which is consistent with the finding that these areas had long arrows on the map showing the direction and distance of the pTOS-induced movement (Fig. $1 F)$. In addition, the map of the distance from the initial to the end point of the forepaw divided by the path length ("straightness index"; Fig. 2C,D; see Materials and Methods) was similar to that of the discreteness index, indicating that most movements with a single velocity peak from an initial posture to a final posture had approximately straight trajectories. It is unlikely that movements with a high discreteness index included rhythmic movements that had a high initial velocity and gradually entered a circular orbit. These results indicate that the discrete movement was represented by domains including the ALM and the HA.

Next, we quantified two aspects of the rhythmic movements. First, for each pTOS site, the frequency and power of the forepaw trajectory was calculated. The map of the frequency with the maximum power showed that high-frequency $(8-10 \mathrm{~Hz})$ movements were generated by pTOS of the CFA (Fig. $2 E$ ). The sites that elicited a movement trajectory with a high magnitude of normalized power at 5-15 Hz (normalized power, $>0.4$ ) were located in the CFA and a medial part of the RFA (Fig. 2F). Second, the rotation direction and total rotation angle of the movement were used to determine the "rotation index" (Fig. 2G,H). A negative rotation index indicates clockwise (i.e., forward) rotation of the right forepaw when viewed from the right side. Most $(76,94$, and $81 \%$ in three mice) of the sites with normalized 5-15 $\mathrm{Hz}$ power $>0.4$ had negative rotation indices of $<-2 \pi$ (Fig. $2 F, H)$. A rotation index of $<-2 \pi$ means that the movement consisted of $>1$ cycle, as shown in Figure $1 E$. Thus, in the domain with high $5-15 \mathrm{~Hz}$ power, the movement was oscillatory and repetitive, i.e., rhythmic. No domain induced a movement with a large positive rotation index (Fig. $2 H$ ). These results indicate that rhythmic movement with forward rotation was represented by the domain spanning from the medial part of the RFA to the CFA. The normalized $5-15 \mathrm{~Hz}$ power of the trajectory is referred to as the "rhythmicity index."

As expected from the finding that the end point of the rhythmic movement varied across trials, the domain with the high rhythmicity index corresponded to a site with relatively short arrows on the map showing the direction and distance of movement (Fig. 1F). Overlying the maps of discreteness and rhythmicity indices reveals clear topography in the broad cortical area in all three mice (Fig. 2I). The domain to induce the rhythmic movement was sandwiched by two domains to induce the discrete movements in forward and backward directions (forward discrete movement and backward discrete movement; Fig. 2I,J). The two domains that induced the forward discrete movement and the rhythmic movement included the lateral agranular cortex and medial agranular cortex and did not include most of the sensory cortex (Tennant et al., 2011; Kirkcaldie, 2012; Figs. 1A, $2 J)$. We confirmed that the discrete and rhythmic movements were reproduced by electrical stimulation of the ALM and CFA, respectively ( $n=2$ mice for each movement type). In the following analyses, we focused on the ALM as the representative area for the forward discrete movement and the CFA as the representative area for the rhythmic movement with forward rotation. We refer 
A

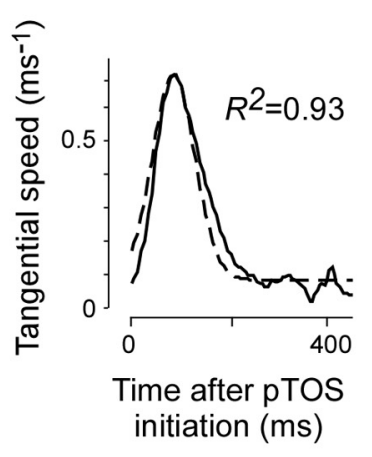

B

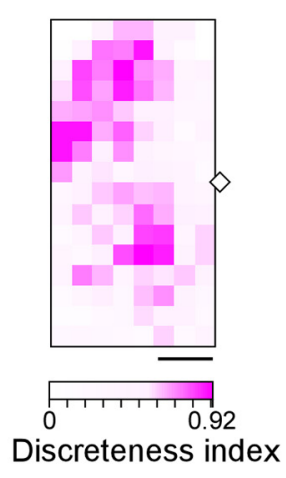

C

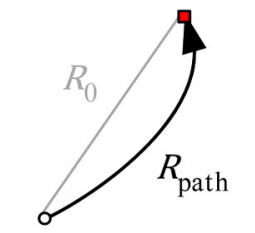

Straightness index $=$

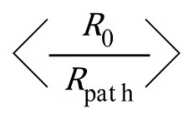

H
E

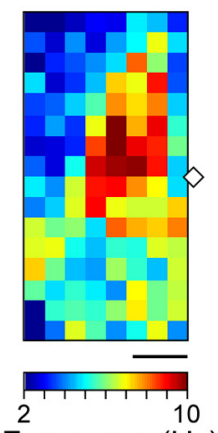

Frequency $(\mathrm{Hz})$
$\mathbf{F}$

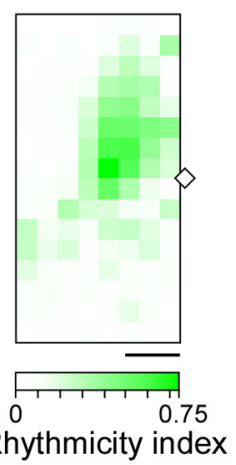

G
D

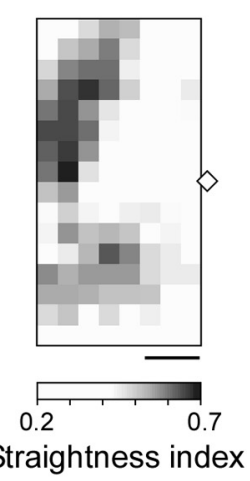

I

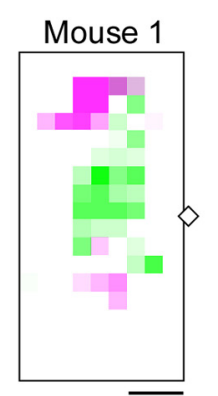

$0.5 \rightleftharpoons 1$

Rhythmicity index
Mouse 2

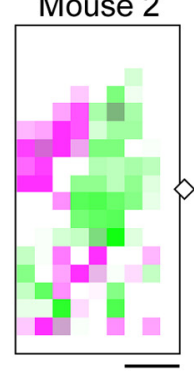

0.8

Discretenss index

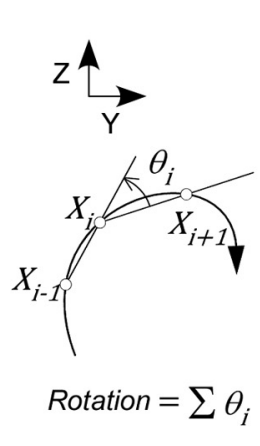

J

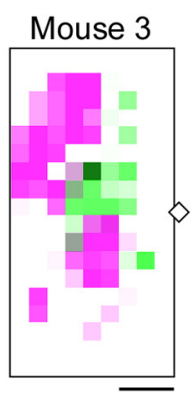

1

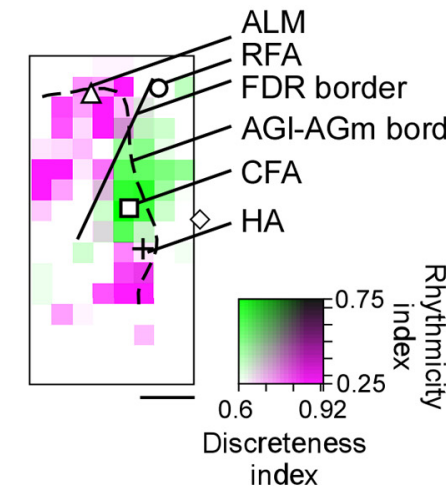

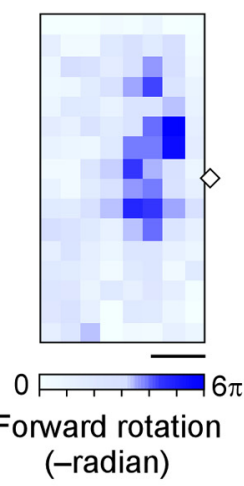

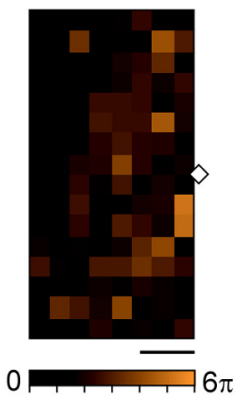

Backward rotation (radian)

Figure 2. Quantitative mapping of discrete and rhythmic movements. $A$, Tangential velocity profile of the mean trajectory shown in Figure $1 D$ (thick line) and the Gaussian function fitted to this trajectory (dotted line). $\boldsymbol{B}$, Map showing the discreteness index for each pTOS site within the square shown in Figure $1 A$, averaged across three mice. The diamond indicates the bregma. Scale bar, $1 \mathrm{~mm}$. C, Definition of the straightness index. D, Map showing the straightness index for each photostimulation site (average of 3 mice). Angle brackets denote trial average. Scale bar, $1 \mathrm{~mm}$. $\boldsymbol{E}$, Map of the maximum frequency of forelimb movements for each pTOS site within the square shown in Figure $1 A$, averaged across three mice. $F$, Map showing the rhythmicity index for each pTOS site, averaged across three mice. $\boldsymbol{G}$, Rotation angle at time $i\left(\theta_{j}\right)$ was calculated using the position of right forepaw observed from the right side of the mouse at three successive time points $\left(X_{i}-{ }_{1}, X_{i}\right.$ and $X_{i+1}$ ). Successive time points were separated by $10 \mathrm{~ms}$. $\theta_{i}$ ranged from $-\pi$ to $\pi$, and forward rotation had a negative value. $\boldsymbol{H}$, Forward (left) and backward (right) rotation angles were mapped. Scale bar, $1 \mathrm{~mm}$. I, Merged maps of discreteness and rhythmicity indices for each of the three mice. $J$, Left, Map of the discreteness index (magenta, $\boldsymbol{B}$ ) overlaid on the map of the rhythmicity index $($ green, $\boldsymbol{F}$ ). The solid line indicates the FDR border. The dotted line indicates the cytoarchitectural border between the lateral agranular cortex and medial agranular cortex (Kirkcaldie, 2012). The triangle, circle, square, and cross indicate the center of the ALM, RFA, CFA, and HA, respectively. Right, Map of the discreteness index overlaid on the map of the rhythmicity index, with the direction and distance of the movement along the $x$-axis and $y$-axis indicated by arrows (Fig. 1F, left).

to the border between these domains as the frontal discreterhythmic movement (FDR) border (Fig. 2J).

\section{Distinct cortical modules for discrete and rhythmic movements}

The optimal photostimulation frequency required to induce discrete movement by pTOS of the ALM was significantly lower than that required to induce rhythmic movement by
pTOS of the CFA (Fig. $3 A, B$ ). The difference in optimal photostimulation frequency was not due to differences in neuronal responses to the photostimulation between the ALM and the CFA (Fig. 3C). When the photostimulation frequency was optimal, the time to reach maximum tangential velocity in the discrete movement was $136 \pm 43 \mathrm{~ms}(n=4$ mice $)$ and the frequency with the maximum power of the rhythmic movement was $8.7 \pm 0.28 \mathrm{~Hz}(n=3$ mice $)$. It was not possible to 
A

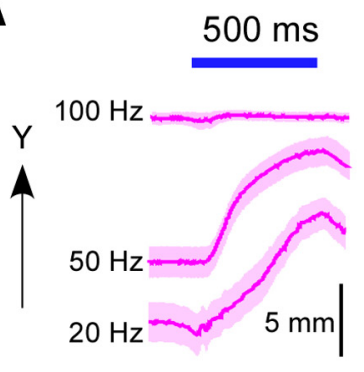

C

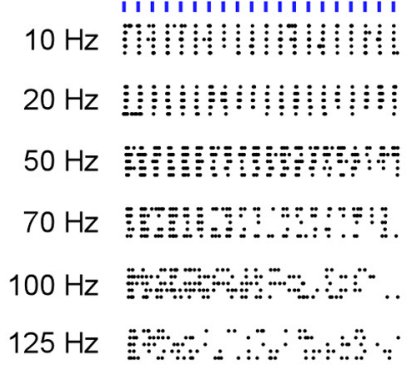

B

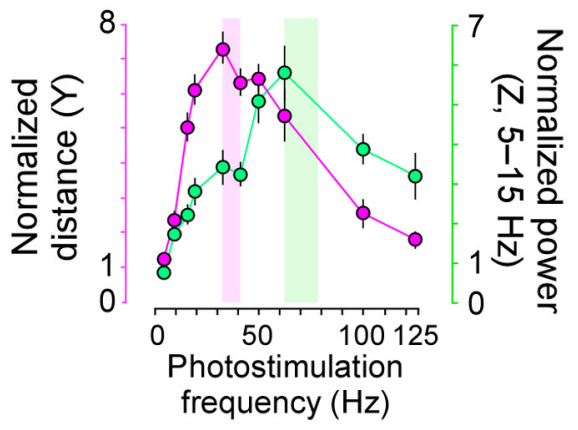

Figure 3. Dependence of the discrete and rhythmic movements on photostimulation frequency. $\boldsymbol{A}$, Left, Time course of the $y$-axis movement induced by $\mathrm{pTOS}$ of the ALM (2.1 mm anterior and $1.9 \mathrm{~mm}$ lateral to bregma). Each line is the average of 20 trials. For each line, pTOS frequency is shown on the left. Right, Time course of the $z$-axis movement induced by pTOS of the CFA ( $0.1 \mathrm{~mm}$ anterior and $1.1 \mathrm{~mm}$ lateral to bregma). Representative trials are shown. $\boldsymbol{B}$, Size (i.e., the distance and the power) of the movement induced by $\mathrm{pTOS}$ of the ALM (magenta) and the (FA (green) as a function of photostimulation frequency. Size was normalized to the size of spontaneous movements in each animal. Magenta-shaded and green-shaded areas indicate the $95 \%$ confidence interval of the optimal frequency estimated by bootstrapping (32.5-40.3 Hz for the discrete movement and $60.8-78.6 \mathrm{~Hz}$ for the rhythmic movement). C, Left, Representative raster plots of spiking activities in the CFA induced by photostimulation ( 20 of $4 \mathrm{~ms}$ pulses) of the CFA at the frequency indicated on the left of each plot. Time scales have been expanded so that the photostimulation timing was aligned for different frequencies. Right, Success rate of induction of spiking activities as a function of the photostimulation frequency. When the spiking was observed for 6 ms after the photostimulation, the photostimulation was regarded as successful.

A

\section{Normalized distance $(Y)$}

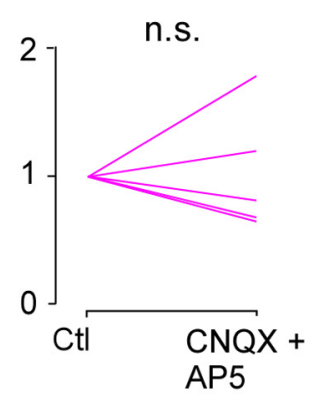

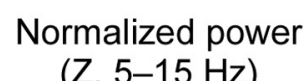

$(\mathrm{Z}, 5-15 \mathrm{~Hz})$

n.s.

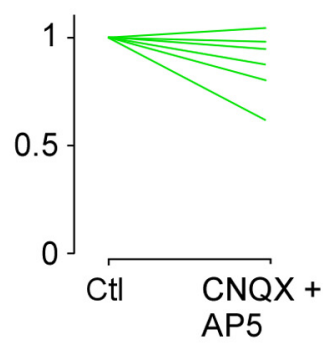

B

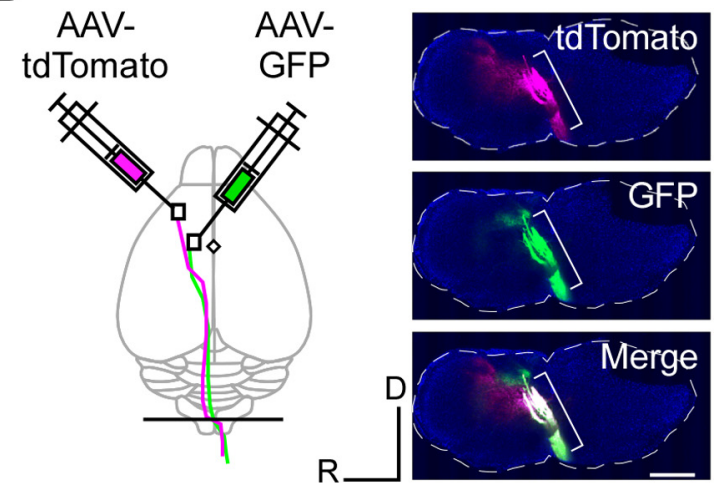

Figure 4. Functional synaptic interaction and anatomical tracing. $A$, Size of the discrete (left) and rhythmic (right) movements before and after bath application of CNQX and D-AP5 (left, $p=0.81$; 5 sites from 2 mice; right, $p=0.094$; 6 sites from 2 mice, Wilcoxon's signed-rank test). $\boldsymbol{B}$, Left, Schematic of simultaneous anterograde tracings from two sites (ALM, magenta, tdTomato; CFA, green, EGFP). The black line denotes the sectioning level for the right panel. Right, Fluorescence images of pyramidal decussation in the coronal section of the medulla shown in left panel. Axons from the ALM (top, magenta) and the CFA (middle, green) were observed. Bracket, Pyramidal decussation. The dotted line indicates the outline of medulla. Scale bar, $1 \mathrm{~mm}$.

predict these values from the optimal photostimulation frequencies. Thus, low-frequency photostimulation of the ALM generated a discrete movement with an intrinsic bell-shaped velocity profile, whereas high-frequency photostimulation of the CFA generated a rhythmic movement with a relatively stable, intrinsic resonance property.

To determine whether the cortical domains to induce the discrete and rhythmic movements were hierarchical or parallel, we applied a mixture of AMPA/kainate receptor antagonist $(2 \mathrm{mM}$ $\mathrm{CNQX}$ ) and NMDA receptor antagonist (2 mM D-AP5) to the cerebral cortex (Hira et al., 2013a). The size of the movement induced by pTOS of the ALM or CFA was not significantly reduced after the application of the blockers to the photostimulated areas (Fig. 4A). Consistently, both the ALM and the CFA sent corticospinal projections through the pyramidal decussations (Fig. 4B). In addition, unsupervised clustering of the sites with corticocortical synaptic interactions obtained by the Allen Mouse Brain Connectivity Atlas (Oh et al., 2014; see Materials and Methods) showed that the border separating two different clusters in the frontal cortex roughly corresponded to the FDR border (Fig. 
$5 A, B)$. Thus, the domains to induce the forward discrete movement and the rhythmic movement were functionally and anatomically distinct. We call such domains "modules."

Nevertheless, there may be polysynaptic interactions between these modules. As predicted, photostimulation of either of the CFA or ALM suppressed spontaneous activity at the other site (Fig. 6A). By contrast, photostimulation of the medial part of the RFA increased activity in the CFA, which is consistent with the finding that these sites are included in the same cluster of synaptic connections. To investigate the possibility that the inhibitory effect between the two modules could weaken the movement induced from the other module, we simultaneously photostimulated the ALM and CFA sites. The distance of the discrete movement induced by the simultaneous pTOS was significantly shorter than that induced by pTOS of the ALM alone (Fig. 6B, top). Likewise, the power of the rhythmic movement was significantly smaller than that induced by pTOS of the CFA alone (Fig. $6 B$, bottom). Thus, the two distinct modules possess the ability to mutually weaken the neuronal activity and the movement induction elicited by the other module.

\section{Ethologically relevant movements} induced by pTOS

Discrete and rhythmic movements might be a part of coordinated movements that involve multiple parts of the body. To relate the discrete and rhythmic modules to the domains involved in coordinated movements, we extracted three types of ethologically relevant movements by detecting movements of other parts of the body as well as the right forelimb. These were termed forepaw-to-mouth, defensive-like, and locomotion-like movements (Fig. $7 A-C$; see Materials and Methods). These movements were reproducibly observed in all animals examined ( $n=4$ mice). Forepaw-to-mouth and locomotion-like movements were mainly elicited by pTOS of the ALM and the CFA, respectively (Fig. 7D). Defensive-like movement was induced by pTOS of the anterior and middle parts of the rhythmic module (Fig. 7D). The border between forepaw-to-mouth and defensive-like movements was almost identical to the FDR border. These results are consistent with the observation that the forepaw-tomouth movement had a constant goal position and that defensive-like and locomotion-like movements included oscillatory components.
A
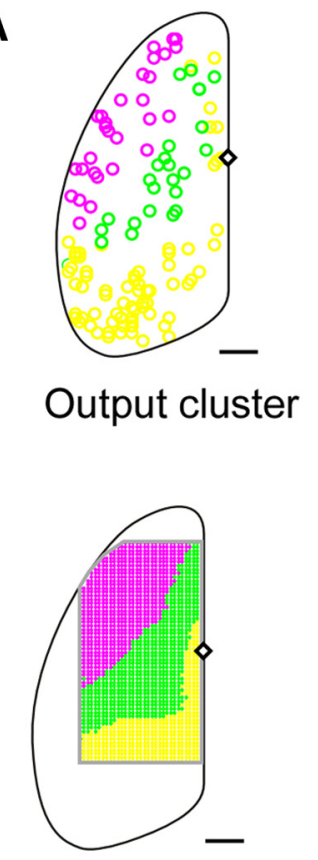

Input cluster
B
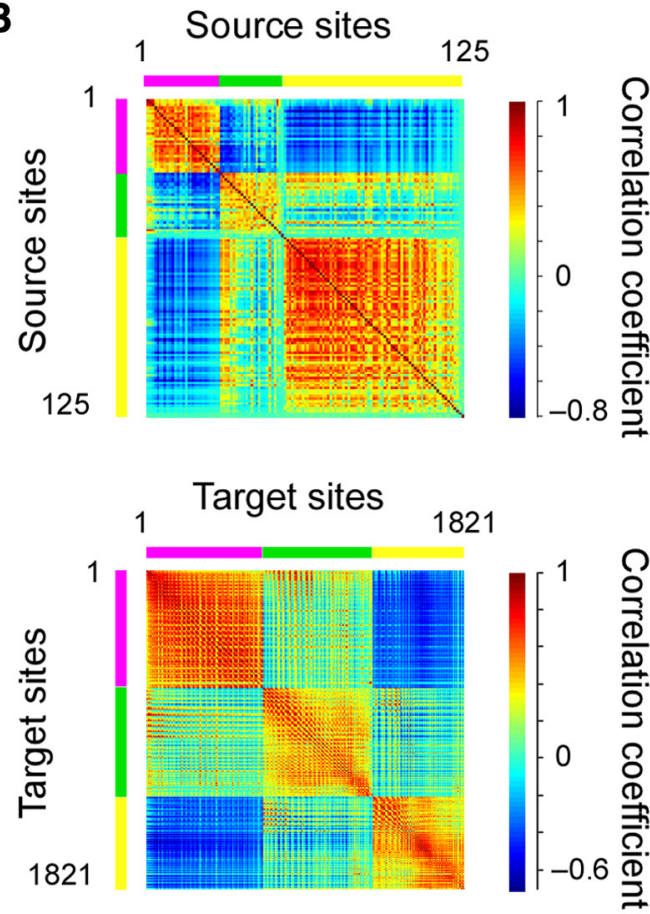

Figure 5. Unsupervised clustering of corticocortical synaptic connections. $\boldsymbol{A}$, Top, Circles indicate the 125 injection sites (source) of anterograde tracer, AAV-EGFP, within the isocortex (Allen Mouse Connectivity Atlas). Bottom, Grids indicate the 1821 target sites for virtual retrograde tracing. The circles and grids with the same color share corticocortical synaptic output (top) and input (bottom) patterns. Scale bar, $1 \mathrm{~mm}$. B, Top, Correlation matrix of all source (injection) sites. Each of three colored clusters corresponded to the same colored sites in $\boldsymbol{A}$ (top). Bottom, Correlation matrix of all target sites. Each of three colored clusters corresponded to the same colored sites in $\boldsymbol{A}$ (bottom).
A
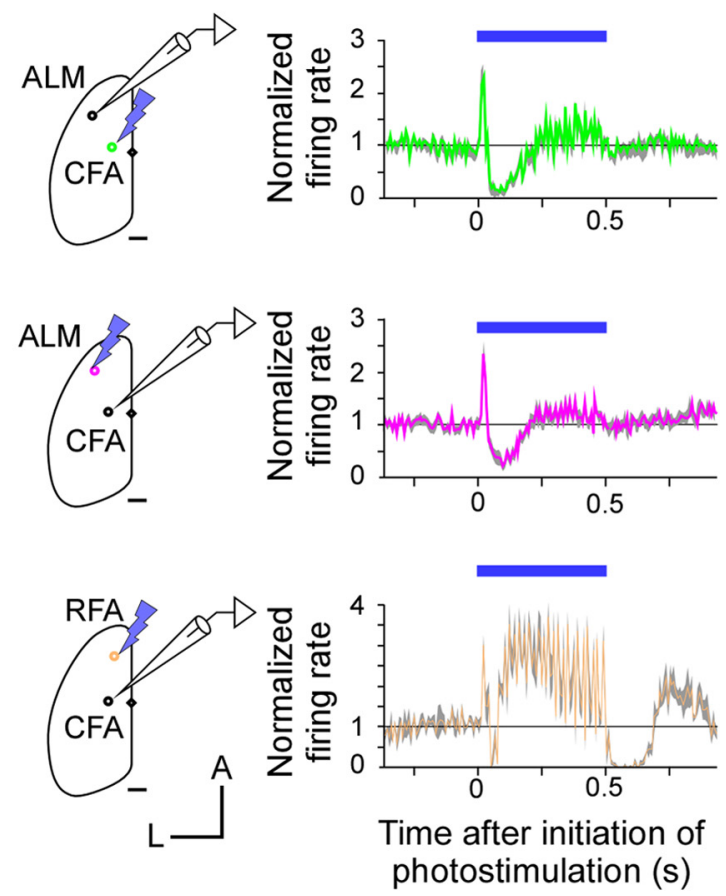

B

Normalized distance $(Y)$

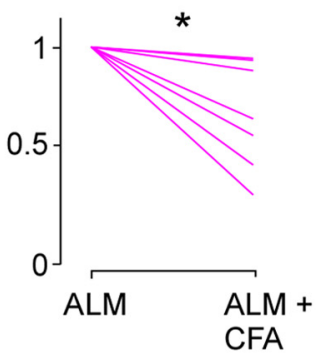

Normalized power $(\mathrm{Z}, 5-15 \mathrm{~Hz})$

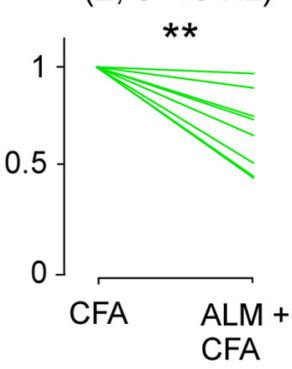

Figure 6. Mutual suppressions of the spontaneous spiking activity and movement induction. $\boldsymbol{A}$, Left, Schematic of simultaneous photostimulation and electrical recording. Scale bar, $1 \mathrm{~mm}$. Right, Normalized firing rate of multiunit activities before, during, and after pTOS. Photostimulation and recording sites are illustrated in the panels on the left. For each experiment, $20-30$ trials were collected from two mice, normalized to the spontaneous firing rate, and then averaged. $\boldsymbol{B}$, Effect of simultaneous pTOS of two sites on the size of the movement. Top, ${ }^{*} p=0.016, n=7$ from two mice. Bottom, ${ }^{* *} p=0.0078, n=8$ from two mice; Wilcoxon's signed-rank test. 
A

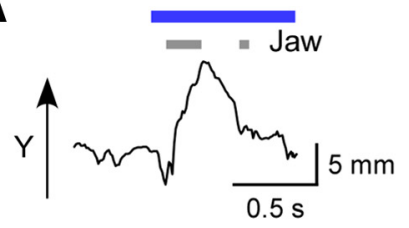

B

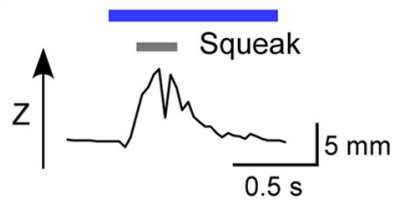

c

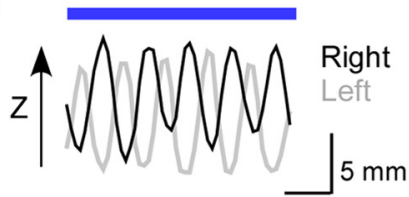

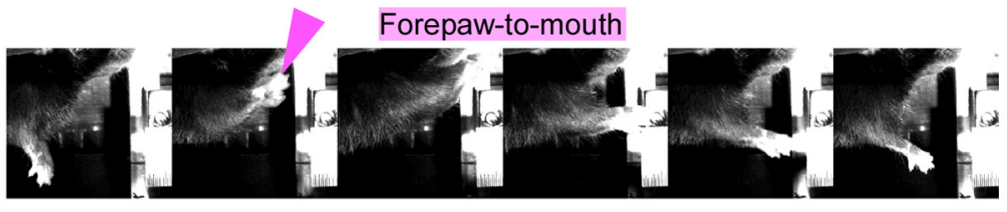

Defensive-like

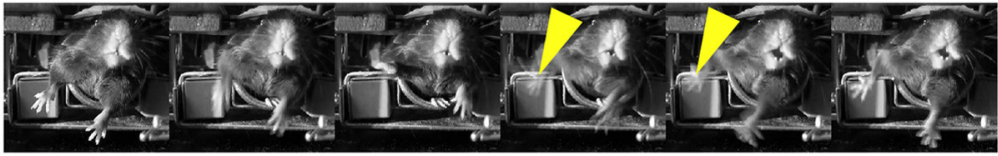

$0.1 \mathrm{~s}$

D

E

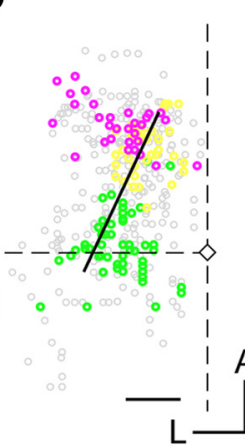

G

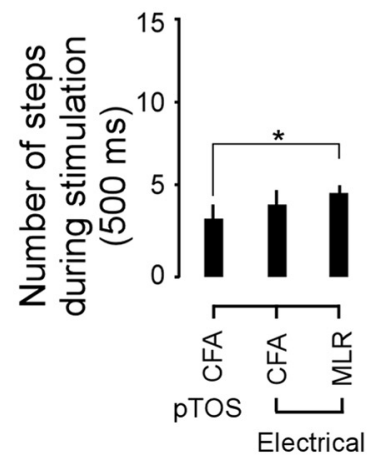

H

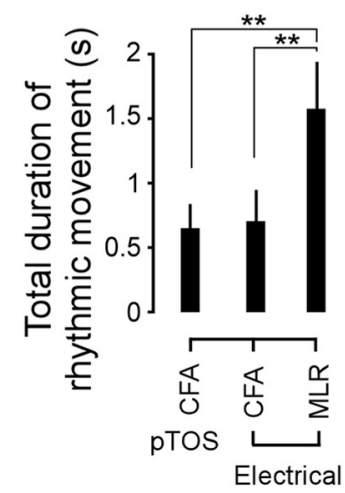

Locomotion-like

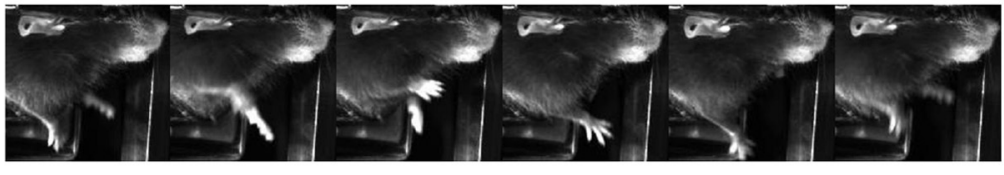

$\mathbf{F}$

Merged
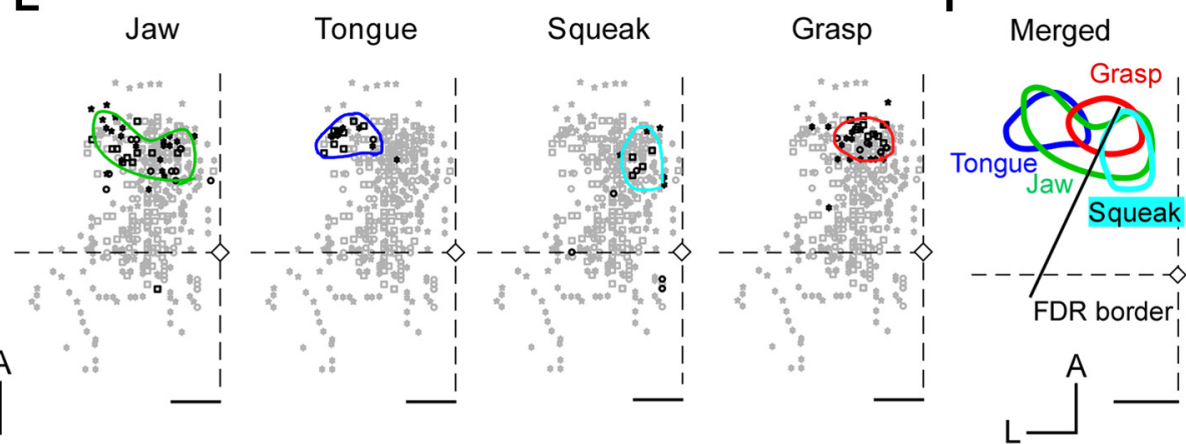

I

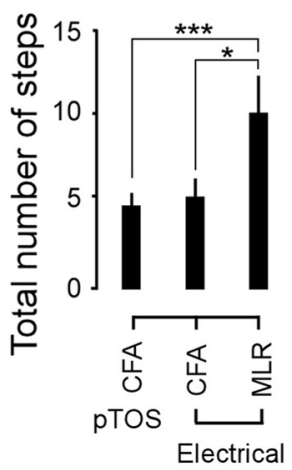

Figure 7. Ethologically relevant movements induced by pTOS. A, Left, Time course of right-forepaw position along the $y$-axis and timing of jaw movement (gray) during a forepaw-to-mouth movement induced by pTOS of the ALM ( $2.4 \mathrm{~mm}$ anterior and $1.5 \mathrm{~mm}$ lateral to bregma). Right, Example images from the video recording during a forepaw-to-mouth movement. The image on the left was obtained at the initiation of the photostimulation and the other images are shown in steps of $100 \mathrm{~ms}$. Magenta arrowhead indicates the right forepaw touching the mouth. The mouth simultaneously opened. $\boldsymbol{B}$, Left, Time course of right-forepaw position along the $z$-axis and timing of squeaking (gray) during a defensive-like movement induced by pTOS ( $1.8 \mathrm{~mm}$ anterior and 1.3 $\mathrm{mm}$ lateral to bregma). Right, Example images from the video recording during a defensive-like movement. The image on the left was obtained at the initiation of the photostimulation and the other images are shown in steps of $100 \mathrm{~ms}$. Green arrowheads indicate afterimages of the right-forelimb oscillatory movement. C, Left, Time course of right-forepaw (black) and left-forepaw (gray) positions in the $z$-axis during a locomotion-like movement induced by pTOS of the CFA ( $0.1 \mathrm{~mm}$ posterior and $1.2 \mathrm{~mm}$ lateral to bregma). Right, Example images from the video recording during a locomotion-like movement. The images were obtained during the photostimulation and are shown in steps of $20 \mathrm{~ms}$. D, Map of three ethologically relevant movements induced by pTOS. Magenta, green, and yellow indicate forepaw-to-mouth, defensive-like, and locomotion-like movements, respectively. Data from eight hemispheres from four mice are overlaid (with 4 maps of right hemispheres mirror reversed). Gray dots denote the sites where pTOS induced other movements or failed to induce any movement. The diamond indicates the bregma. Scale bar, $1 \mathrm{~mm}$. $\boldsymbol{E}$, Maps of the jaw movements, tongue movements (licking), human-audible squeaks, and grasping. The four types of symbols indicate data from four different mice. The overlaid contour lines show the approximate outlines of the cortical areas representing the movement types. $\boldsymbol{F}$, Merged map of the contour lines shown in $\boldsymbol{E}$. The FDR border (black line) is overlaid. The diamond indicates bregma. Scale bar, $1 \mathrm{~mm}$. G, Number of steps made by the right forepaw during $500 \mathrm{~ms}$ pTOS of the CFA, $500 \mathrm{~ms}$ electrical stimulation of the CFA, and $500 \mathrm{~ms}$ electrical stimulation of the MLR (ANOVA, $p=$ 0.0045 ; post hoc Tukey's HSD test, ${ }^{*} p<0.05$ ). $\boldsymbol{H}$, The total duration of the rhythmic movements induced by pTOS of the CFA, electrical stimulation of the CFA, and electrical stimulation of the MLR (ANOVA, $p=0.0013$; post hoc Tukey's HSD test, ${ }^{* *} p<0.01$ ). II, Total number of steps made by the right forepaw per pTOS of the CFA, electrical stimulation of the CFA, and electrical stimulation of the MLR (ANOVA, $p=0.0001$; post hoc Tukey's HSD test, ${ }^{* * *} p<10^{-4},{ }^{*} p<0.05$ ). 
A

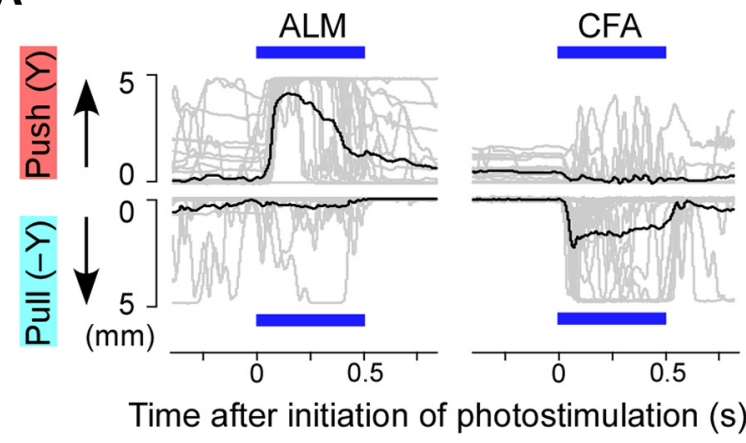

B

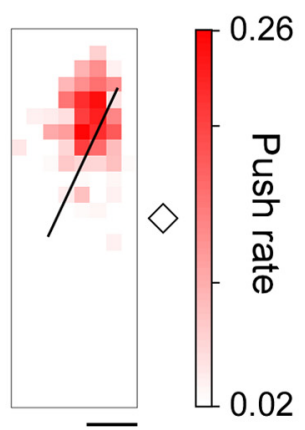

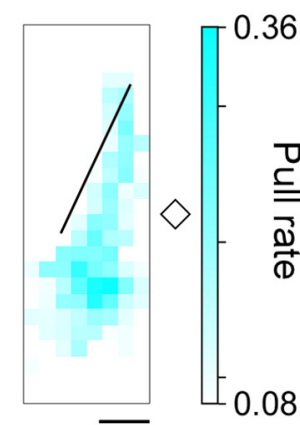

Figure 8. Lever-push and lever-pull movements induced by pTOS. A, Lever-push (top) and lever-pull (bottom) movements induced by pTOS of the ALM (left, $2.1 \mathrm{~mm}$ anterior and $1.9 \mathrm{~mm}$ lateral to bregma) and (FA (right, $0.2 \mathrm{~mm}$ posterior and $1.2 \mathrm{~mm}$ lateral to bregma). Gray traces represent individual lever trajectories (20 trials). Black traces indicate average lever trajectory. Blue bars denote 500 ms pTOS. B, Maps of lever-push (left, red) and lever-pull (right, cyan) movements induced by pTOS. The map represents the average of four mice. The black line indicates the FDR border. Scale bar, $1 \mathrm{~mm}$.

We also mapped elements of the ethologically relevant movements, and obtained similar results to those observed in studies of rodents (Ramanathan et al., 2006; Komiyama et al., 2010; Bonazzi et al., 2013; Fig. 7 E,F). Licking, which was sometimes observed during forepaw-to-mouth movement, was represented in the discrete module. Squeaking, which often accompanied defensive-like movement, was represented in the rhythmic module. Jaw movement and grasping, which were related to both movements, were represented in both modules. These results suggest that the discrete and rhythmic modules incorporate the representation of elemental movements required for the corresponding ethologically relevant movements.

Furthermore, we determined whether locomotion-like movements induced by the CFA stimulation were similar to movements represented in the MLR (Fouad and Pearson, 1997; Lee et al., 2014). When the number of forelimb steps induced by the electrical stimulation of the CFA was similar to that induced by pTOS of the CFA, the number taken during the electrical stimulation with the identical parameters was similar between the CFA and the MLR, and steps were taken at $\sim 8 \mathrm{~Hz}$ (Fig. $7 G$ ). This stepping frequency was faster than that of walking $(2-5 \mathrm{~Hz}$; Fouad and Pearson, 1997) and presumably corresponded to running (Lee et al., 2014). The limb movement stopped immediately after CFA stimulation, but continued for $\sim 1000 \mathrm{~ms}$ after MLR stimulation (Fig. $7 \mathrm{H}, \mathrm{I}$ ). Bilateral hindlimb movements were always induced by MLR stimulation, but not always induced by CFA stimulation. These results indicate that the CFA is less specialized to locomotion than the MLR.

\section{Induction of lever-push and lever-pull movements by pTOS}

Although the medial parts of RFA and the CFA were each involved in the rhythmic module, neuronal activity in these areas is strongly related to a voluntary lever-pull movement that is not rhythmic (Hira et al., 2013a; Saiki et al., 2014). Therefore, these areas may not function only for rhythmic movement. To assess this possibility, we conducted pTOS when a lever was located near the right forepaw in four mice. As predicted by the finding that pTOS of the ALM induced a forward movement (Fig. 1D), this stimulation exclusively induced a lever-push movement (Fig. $8 A$ ). On the other hand, pTOS of the CFA preferentially induced a lever-pull movement (Fig. $8 A$ ), which was sometimes followed by the rhythmic movement. The lever pushing and pulling started immediately after the onset of $500 \mathrm{~ms}$ pTOS and was maintained for $411 \pm 91 \mathrm{~ms}$ and $312 \pm 139 \mathrm{~ms}$, respectively. The medial parts of RFA and the HA almost exclusively induced a lever-pull movement. The border of the lever-push and lever-pull movements roughly corresponded to the FDR border (Fig. 8B). These results indicate that the distinct cortical modules adapted to distinct lever movements, and that the module for the rhythmic movement could induce a nonrhythmic movement.

\section{Discussion}

To our knowledge, the present study is the first to map the discrete and rhythmic forelimb movements, including locomotionlike and defensive-like forelimb movements and lever-push and lever-pull movements, in the rodent cortex. Most mapping experiments so far have used anesthetized rodents, in which natural behaviors were substantially suppressed (Ramanathan et al., 2006; Hira et al., 2009; Harrison et al., 2012; Bonazzi et al., 2013; Silasi et al., 2013; Brown and Teskey, 2014), because it has been difficult to stably monitor forelimb movements in awake ones. By contrast, in the present study, awake, head-fixed mice were accustomed to a condition where their forelimbs were off the ground. After habituation to the experimental condition, the mice were relaxed and maintained the natural posture without their forelimbs touching the ground. This might explain why complex forelimb movements were successfully induced, as they have been in awake monkeys (Graziano et al., 2002; Stepniewska et al., 2005).

We identified an area of the cerebral cortex consisting of a rhythmic movement domain sandwiched between two domains for forward and backward discrete movements. The cortical modules for the forward discrete movement and the rhythmic movement had different optimal stimulation frequencies for induction of movements and belonged to distinct clusters of corticocortical synaptic circuits. They mutually inhibited each other's neuronal activity, and independently had corticospinal projections. The border of these modules, termed the FDR border, also discriminated the representation of ethologically relevant movements and lever-push/pull movements. Our results extend the notion that the motor cortex consists of functionally and anatomically divided modules (Huntley and Jones, 1991; Capaday et al., 2009). The FDR border was orthogonal to the histological border between the lateral agranular cortex and medial agranular cortex, which is thought to be a border between lower-order and higher-order motor cortices (Sul et al., 2011). Hierarchical interactions might be present within each module (Smith et al., 2010; Ueta et al., 2014). Future studies should evaluate whether the modular organization of the motor cortex with different cyto- 
architectures is evolutionally conserved across mammalian species.

The frequency of the rhythmic movement elicited by pTOS of the CFA was relatively stable, at $8-10 \mathrm{~Hz}$. This is similar to the frequency range of whisking and licking (Haiss and Schwarz, 2005; Komiyama et al., 2010), suggesting that the rhythmic movement may have an intrinsic resonant property whatever muscles are targeted. By contrast, the time to reach maximum velocity in the discrete movement corresponded to approximately one-fourth of a $2 \mathrm{~Hz}$ oscillation, which is much less than the frequency of rhythmic movement. Thus, the present study supports the hypothesis that rhythmic movements are not repeated discrete movements (Schaal et al., 2004; Hogan and Sternad, 2007; Degallier and Ijspeert, 2010; Howard et al., 2011). However, when a lever was located near the forepaw, pTOS of the domain for the rhythmic movement induced a lever-pull movement that was not rhythmic. The lever pull was maintained for $>300 \mathrm{~ms}$, which was longer than the duration of half a cycle of the rhythmic movement. It is possible to interpret these findings as indicating that the first half-cycle of the rhythmic movement can be adapted to a unidirectional discrete movement, as previously proposed (Degallier and Ijspeert, 2010; Howard et al., 2011), and can be prolonged by feedback from the forelimb (Graziano, 2006).

Thus, motor cortical neurons may not exclusively encode unique purposes or specific complex movements (Scott, 2008; Shenoy et al., 2013). Instead, a population of neurons in a motor cortical area may evolve to encode various purposeful movements, depending on the environmental constraints and intrinsic dynamics. It is still unknown how the complex forelimb movements induced by pTOS are volitionally generated. To elucidate the computations in individual cortical areas and the relation among cortical networks for purposeful movements, it is necessary to compare neuronal activity in multiple cortical modules when voluntary behaviors are adapted to changing needs (Hira et al., 2014).

\section{References}

Alstermark B, Isa T (2012) Circuits for skilled reaching and grasping. Annu Rev Neurosci 35:559-578. CrossRef Medline

Asrican B, Augustine GJ, Berglund K, Chen S, Chow N, Deisseroth K, Feng G, Gloss B, Hira R, Hoffmann C, Kasai H, Katarya M, Kim J, Kudolo J, Lee LM, Lo SQ, Mancuso J, Matsuzaki M, Nakajima R, Qiu L, et al. (2013) Next-generation transgenic mice for optogenetic analysis of neural circuits. Front Neural Circuits 7:160. CrossRef Medline

Ayling OG, Harrison TC, Boyd JD, Goroshkov A, Murphy TH (2009) Automated light-based mapping of motor cortex by photoactivation of channelrhodopsin-2 transgenic mice. Nat Methods 6:219-224. CrossRef Medline

Bartolomucci A, Pederzani T, Sacerdote P, Panerai AE, Parmigiani S, Palanza P (2004) Behavioral and physiological characterization of male mice under chronic psychosocial stress. Psychoneuroendocrinology 29:899-910. CrossRef Medline

Beloozerova IN, Sirota MG (1993) The role of the motor cortex in the control of accuracy of locomotor movements in the cat. J Physiol 461:1-25. CrossRef Medline

Bonazzi L, Viaro R, Lodi E, Canto R, Bonifazzi C, Franchi G (2013) Complex movement topography and extrinsic space representation in the rat forelimb motor cortex as defined by long-duration intracortical microstimulation. J Neurosci 33:2097-2107. CrossRef Medline

Brown AR, Teskey GC (2014) Motor cortex is functionally organized as a set of spatially distinct representations for complex movements. J Neurosci 34:13574-13585. CrossRef Medline

Capaday C, Ethier C, Brizzi L, Sik A, van Vreeswijk C, Gingras D (2009) On the nature of the intrinsic connectivity of the cat motor cortex: evidence for a recurrent neural network topology. J Neurophysiol 102:2131-2141. CrossRef Medline
Cooke DF, Graziano MS (2004) Sensorimotor integration in the precentral gyrus: polysensory neurons and defensive movements. J Neurophysiol 91:1648-1660. CrossRef Medline

Degallier S, Ijspeert A (2010) Modeling discrete and rhythmic movements through motor primitives: a review. Biol Cybern 103:319-338. CrossRef Medline

Fouad K, Pearson KG (1997) Effects of extensor muscle afferents on the timing of locomotor activity during walking in adult rats. Brain Res 749: 320-328. CrossRef Medline

Georgopoulos A, Grillner S (1989) Visuomotor coordination in reaching and locomotion. Science 245:1209-1210. CrossRef Medline

Graziano M (2006) The organization of behavioral repertoire in motor cortex. Annu Rev Neurosci 29:105-134. CrossRef Medline

Graziano MS, Taylor CS, Moore T (2002) Complex movements evoked by microstimulation of precentral cortex. Neuron 34:841-851. CrossRef Medline

Haiss F, Schwarz C (2005) Spatial segregation of different modes of movement control in the whisker representation of rat primary motor cortex. J Neurosci 25:1579-1587. CrossRef Medline

Harrison TC, Ayling OG, Murphy TH (2012) Distinct cortical circuit mechanisms for complex forelimb movement and motor map topography. Neuron 74:397-409. CrossRef Medline

Hira R, Honkura N, Noguchi J, Maruyama Y, Augustine GJ, Kasai H, Matsuzaki M (2009) Transcranial optogenetic stimulation for functional mapping of the motor cortex. J Neurosci Methods 179:258-263. CrossRef Medline

Hira R, Ohkubo F, Ozawa K, Isomura Y, Kitamura K, Kano M, Kasai H, Matsuzaki M (2013a) Spatiotemporal dynamics of functional clusters of neurons in the mouse motor cortex during a voluntary movement. J Neurosci 33:1377-1390. CrossRef Medline

Hira R, Ohkubo F, Tanaka YR, Masamizu Y, Augustine GJ, Kasai H, Matsuzaki M (2013b) In vivo optogenetic tracing of functional corticocortical connections between motor forelimb areas. Front Neural Circuits 7:55. CrossRef Medline

Hira R, Ohkubo F, Masamizu Y, Ohkura M, Nakai J, Okada T, Matsuzaki M (2014) Reward-timing-dependent bidirectional modulation of cortical microcircuits during optical single-neuron operant conditioning. Nat Commun 5:5551. CrossRef Medline

Hogan N, Sternad D (2007) On rhythmic and discrete movements: reflections, definitions and implications for motor control. Exp Brain Res 181: 13-30. CrossRef Medline

Howard IS, Ingram JN, Wolpert DM (2011) Separate representations of dynamics in rhythmic and discrete movements: evidence from motor learning. J Neurophysiol 105:1722-1731. CrossRef Medline

Huntley GW, Jones EG (1991) Relationship of intrinsic connections to forelimb movement representations in monkey motor cortex: a correlative anatomic and physiological study. J Neurophysiol 66:390-413. Medline

Iwaniuk AN, Whishaw IQ (2000) On the origin of skilled forelimb movements. Trends Neurosci 23:372-376. CrossRef Medline

Kirkcaldie MTK (2012) Neocortex. In: The mouse nervous system. (Watson C, Paxinos G, Puelles L, eds), pp 52-111. San Diego: Academic.

Komiyama T, Sato TR, O'Connor DH, Zhang YX, Huber D, Hooks BM, Gabitto M, Svoboda K (2010) Learning-related fine-scale specificity imaged in motor cortex circuits of behaving mice. Nature 464:1182-1186. CrossRef Medline

Lee AM, Hoy JL, Bonci A, Wilbrecht L, Stryker MP, Niell CM (2014) Identification of a brainstem circuit regulating visual cortical state in parallel with locomotion. Neuron 83:455-466. CrossRef Medline

Masamizu Y, Tanaka YR, Tanaka YH, Hira R, Ohkubo F, Kitamura K, Isomura Y, Okada T, Matsuzaki M (2014) Two distinct layer-specific dynamics of cortical ensembles during learning of a motor task. Nat Neurosci 17:987-994. CrossRef Medline

Morasso P (1981) Spatial control of arm movements. Exp Brain Res 42:223227. Medline

Oh SW, Harris JA, Ng L, Winslow B, Cain N, Mihalas S, Wang Q, Lau C, Kuan L, Henry AM, Mortrud MT, Ouellette B, Nguyen TN, Sorensen SA, Slaughterbeck CR, Wakeman W, Li Y, Feng D, Ho A, Nicholas E, et al. (2014) A mesoscale connectome of the mouse brain. Nature 508:207214. CrossRef Medline

Ramanathan D, Conner JM, Tuszynski MH (2006) A form of motor cortical plasticity that correlates with recovery of function after brain injury. Proc Natl Acad Sci U S A 103:11370-11375. CrossRef Medline 
Saiki A, Kimura R, Samura T, Fujiwara-Tsukamoto Y, Sakai Y, Isomura Y (2014) Different modulation of common motor information in rat primary and secondary motor cortices. PloS One 9:e98662. CrossRef Medline

Schaal S, Sternad D, Osu R, Kawato M (2004) Rhythmic arm movement is not discrete. Nat Neurosci 7:1136-1143. CrossRef Medline

Scott SH (2008) Inconvenient truths about neural processing in primary motor cortex. J Physiol 586:1217-1224. CrossRef Medline

Shenoy KV, Sahani M, Churchland MM (2013) Cortical control of arm movements: a dynamical systems perspective. Annu Rev Neurosci 36: 337-359. CrossRef Medline

Silasi G, Boyd JD, Ledue J, Murphy TH (2013) Improved methods for chronic light-based motor mapping in mice: automated movement tracking with accelerometers, and chronic EEG recording in a bilateral thinskull preparation. Front Neural Circuits 7:123. CrossRef Medline

Smith NJ, Horst NK, Liu B, Caetano MS, Laubach M (2010) Reversible inactivation of rat premotor cortex impairs temporal preparation, but not inhibitory control, during simple reaction-time performance. Front Integr Neurosci 4:124. Medline

Stepniewska I, Fang PC, Kaas JH (2005) Microstimulation reveals specialized subregions for different complex movements in posterior parietal cortex of prosimian galagos. Proc Natl Acad Sci U S A 102:4878-4883. CrossRef Medline

Sul JH, Jo S, Lee D, Jung MW (2011) Role of rodent secondary motor cortex in value-based action selection. Nat Neurosci 14:1202-1208. CrossRef Medline

Tennant KA, Adkins DL, Donlan NA, Asay AL, Thomas N, Kleim JA, Jones TA (2011) The organization of the forelimb representation of the C57BL/6 mouse motor cortex as defined by intracortical microstimulation and cytoarchitecture. Cereb Cortex 21:865-876. CrossRef Medline

Ueta Y, Otsuka T, Morishima M, Ushimaru M, Kawaguchi Y (2014) Multiple layer 5 pyramidal cell subtypes relay cortical feedback from secondary to primary motor areas in rats. Cereb Cortex 24:2362-2376. CrossRef Medline 